University of New Hampshire

University of New Hampshire Scholars' Repository

Space Science Center

Institute for the Study of Earth, Oceans, and

Space (EOS)

$2-1-2003$

\title{
Hard x-ray polarimetry with the Ramaty High Energy Solar Spectroscopic Imager (RHESSI)
}

\author{
Mark L. McConnell \\ University of New Hampshire - Main Campus, mark.mcconnell@unh.edu \\ David M. Smith \\ University of California - Berkeley \\ A G. Emslie \\ University of Alabama \\ Robert P. Lin \\ University of California - Berkeley \\ James M. Ryan \\ University of New Hampshire, James.Ryan@unh.edu
}

Follow this and additional works at: https://scholars.unh.edu/ssc

Part of the Astrophysics and Astronomy Commons

\section{Recommended Citation}

Mark L. McConnell ; David M. Smith ; A. Gordon Emslie ; Robert P. Lin and James M. Ryan "Hard x-ray polarimetry with the Ramaty High Energy Solar Spectroscopic Imager (RHESSI)", Proc. SPIE 4843, Polarimetry in Astronomy, 8 (February 1, 2003); doi:10.1117/12.458879; http://dx.doi.org/10.1117/ 12.458879

This Conference Proceeding is brought to you for free and open access by the Institute for the Study of Earth, Oceans, and Space (EOS) at University of New Hampshire Scholars' Repository. It has been accepted for inclusion in Space Science Center by an authorized administrator of University of New Hampshire Scholars' Repository. For more information, please contact Scholarly.Communication@unh.edu. 


\title{
Hard X-ray polarimetry with the Ramaty High Energy Solar Spectroscopic Imager (RHESSI)
}

\author{
Mark L. McConnell ${ }^{1}$, David M. Smith ${ }^{2}$, A. Gordon Emslie ${ }^{3}$, \\ Robert P. Lin ${ }^{4}$ and James M. Ryan ${ }^{1}$ \\ ${ }^{1}$ Space Science Center, University of New Hampshire, Durham, NH 03824 \\ ${ }^{2}$ Space Sciences Laboratory, University of California, Berkeley, CA 94720 \\ ${ }^{3}$ Department of Physics, University of Alabama, Huntsville, AL 35899 \\ 4Physics Department and Space Sciences Laboratory, \\ University of California, Berkeley, CA 94720
}

\begin{abstract}
Although designed primarily as a hard X-ray imager and spectrometer, the Ramaty High Energy Solar Spectroscopic Imager (RHESSI) is also capable of measuring the polarization of hard X-rays (20-100 keV) from solar flares. This capability arises from the inclusion of a small unobstructed Be scattering element that is strategically located within the cryostat that houses the array of nine germanium detectors. The Ge detectors are segmented, with both a front and rear active volume. Low energy photons (below about $100 \mathrm{keV}$ ) can reach a rear segment of a Ge detector only indirectly, by scattering. Low energy photons from the Sun have a direct path to the Be and have a high probability of Compton scattering into a rear segment of a Ge detector. The azimuthal distribution of these scattered photons carries with it a signature of the linear polarization of the incident flux. Sensitivity estimates, based on simulations and in-flight background measurements, indicate that a 20-100 keV polarization sensitivity of less than a few percent can be achieved for X-class flares.
\end{abstract}

Keywords: X-ray, X-ray astronomy, gamma-ray gamma-ray astronomy, polarization, polarimetry, Sun, solar flare

\section{INTRODUCTION}

Hard X-ray emission from solar flares, like any other form of electromagnetic radiation, has four, and only four properties. Each photon can be completely characterized by its time of arrival, its energy, its direction of arrival, and its polarization state. RHESSI is capable, to varying degrees, of determining all four of these quantities. The first two of these properties (time of arrival and energy) are measured directly as photon interactions in the Ge detectors. The third and fourth properties (arrival direction and polarization state) are determined through an analysis of the grouping of photons in time at each detector. RHESSI will capable of providing information on the linear polarization of photons between roughly 20 and $100 \mathrm{keV}{ }^{1}$ The study of polarization at hard X-ray energies is especially appealing in that the hard X-ray emission from any bremsstrahlung source (such as a solar flare) will be polarized if the phase-space distribution of the emitting electrons is anisotropic. Polarization measurements therefore provide a direct handle on the extent to which the accelerated electrons are beamed, which, in turn, has important implications for particle acceleration models.

Many models of nonthermal (e.g., thick target) hard X-ray production predict a clear and significant polarization signal, with polarization levels $>10 \% .^{2-8}$ The precise level of polarization depends on both energy and viewing angle. Even thermal models of the hard X-ray source predict a finite polarization of order a few percent, due to the anisotropy in the electron distribution function caused by a thermal conductive flux out of the emitting region into the cooler surroundings. ${ }^{9}$ The thermal component, with its rather low polarization, tends to dominate the emission from all flares at energies below about $25 \mathrm{keV}$. At these energies, it therefore becomes difficult to distinguish the non-thermal component, with its intrinsic directivity signature, from the

Correspondence: E-mail: Mark.McConnell@unh.edu 
thermal component. This has led to the argument that polarization measurements can best be performed at higher energies. ${ }^{10}$

These predictions, while clearly testable, could be criticized on the grounds that the modeling assumptions they contain may be oversimplistic. For example, each model to date assumes a single, simple magnetic field structure about which the emitting electrons spiral. It could be argued that any real flare, particularly one sufficiently large to produce a signal of sufficient strength to enable a polarization measurement to be made, will in all probability contain a mix of structures that would average out any polarization signal present. However, observations from Yohkoh of large, simple magnetic structures even in large flares ${ }^{11}$ give support to the possibility that a statistically significant polarization signal could be produced in a large event.

The first measurements of X-ray polarization from solar flares (at energies of $\sim 15 \mathrm{keV}$ ) were made by Soviet experimenters using polarimeters aboard the Intercosmos satellites. In their initial study, Tindo et al. ${ }^{12}$ reported an average polarization for three small X-ray flares of $\mathrm{P}=40 \%( \pm 20 \%)$. This study was followed by an analysis of three flares in October and November of $1970^{13,14}$ that showed polarizations of approximately $20 \%$ during the hard impulsive phase. These reports were met with considerable skepticism, on the grounds that they did not adequately allow for detector cross-calibration issues and limited photon statistics. ${ }^{15}$ Subsequent observations with an instrument on the OSO-7 satellite seemed to confirm the existence and magnitudes of the polarizations $(\sim 10 \%)$, but these data were compromised by in-flight gain shifts. ${ }^{16}$ In a later study using a polarimeter on Intercosmos 11 , Tindo et al. ${ }^{17}$ measured polarizations of only a few percent at $\sim 15 \mathrm{keV}$ for two flares in July 1974. This small but finite polarization is consistent with the predictions for purely thermal emission that contains an admixture of polarized backscattered radiation. ${ }^{2}$ A small polarimeter was flown on an early shuttle flight (STS-3) ${ }^{18}$ and made measurements of eight C- and M-class flares in the 5-20 keV energy range. Upper limits in the range of $2.5 \%$ to $12.7 \%$ were measured, although contamination of the Li scattering material invalidated the pre-flight calibration. ${ }^{19}$

The polarimetric capability of RHESSI offers a unique opportunity to study solar flares. RHESSI will be able to make polarization measurements at energies higher than previous studies, where thermal emission may be less of an issue. In addition, not only does RHESSI provide significant polarization sensitivity, the capability of RHESSI to simultaneuously image the hard X-ray emission represents a major advantage over previous efforts to measure hard X-ray polarization.

\section{COMPTON POLARIMETERS}

The basic physical process used to measure polarization in the $20-100 \mathrm{keV}$ energy range is Compton scattering, ${ }^{20}$ the cross-section for which is given by, ${ }^{21}$

$$
d \sigma=\frac{r_{0}^{2}}{2} d \Omega\left(\frac{\nu^{\prime}}{\nu_{o}}\right)^{2}\left(\frac{\nu_{o}}{\nu^{\prime}}+\frac{\nu^{\prime}}{\nu_{o}}-2 \sin ^{2} \theta \cos ^{2} \eta\right)
$$

where,

$$
\frac{\nu^{\prime}}{\nu_{o}}=\frac{1}{1+\left(\frac{h \nu_{o}}{m c^{2}}\right)(1-\cos \theta)}
$$

Here $\nu_{o}$ is the frequency of the incident photon, $\nu^{\prime}$ is the frequency of the scattered photon, $\theta$ is the scattering angle of the scattered photon measured from the direction of the incident photon, and $\eta$ is the azimuthal angle of the scattered photon measured from the plane containing the electric vector of the incident photon. For a given value of $\theta$, the scattering cross section for polarized radiation reaches a minimum at $\eta=0^{\circ}$ and a maximum at $\eta=90^{\circ}$. In other words, photons tend to be scattered at a right angle with respect to the incident electric field vector. In the case of an unpolarized beam of incident photons, there will be no net positive electric field vector and therefore no preferred azimuthal scattering angle $(\eta)$; the distribution of scattered photons with respect to $\eta$ will be uniform. However, in the polarized case, the incident photons will exhibit a net positive electric field vector and the distribution in $\eta$ will be asymmetric. 


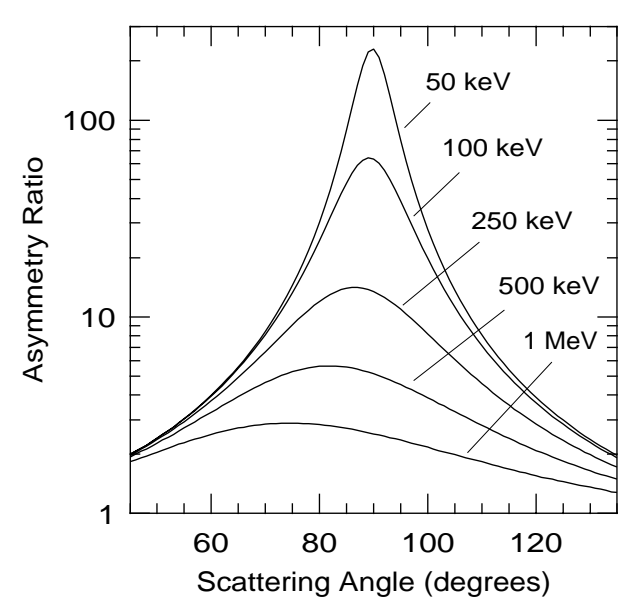

Figure 1: The asymmetry ratio (Eq. 3) for various incident photon energies.

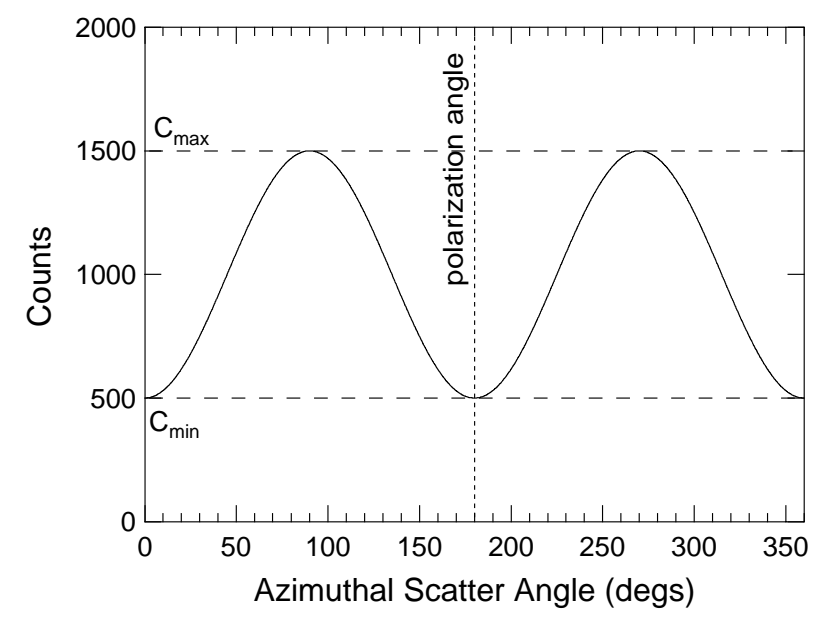

Figure 2: The modulation pattern produced by Compton scattering of polarized radiation. The minimum of the modulation pattern defines the plane of polarization of the incident flux. In this case, $\phi=180^{\circ}$.

The ability to measure the polarization of the incident photon beam depends on the asymmetry ratio. ${ }^{22}$ This is defined to be the ratio between the maximum in the $\eta$ distribution to the minimum intensity in the $\eta$ distribution,

$$
R\left(\frac{\max }{\min }\right)=\frac{\left(\nu_{o} / \nu^{\prime}+\nu^{\prime} / \nu_{o}\right)}{\left(\nu_{o} / \nu^{\prime}+\nu^{\prime} / \nu_{o}-2 \sin ^{2} \theta\right)}
$$

This distribution is plotted as a function of scattering angle $(\theta)$ for various incident photon energies in Fig. 1. This plot shows that the asymmetry ratio is larger at lower energies and that events with scattering angles between $60^{\circ}$ and $120^{\circ}$ contain most of the polarization information.

In general, a Compton scatter polarimeter consists of two detectors to determine the energies of both the scattered photon and the scattered electron. One detector, the scattering detector, provides the medium for the Compton interaction to take place. This detector must be designed to maximize the probability of there being a single Compton interaction with a subsequent escape of the scattered photon. This requires a low- $\mathrm{Z}$ material in order to minimize photoelectric interactions. The area of the scattering detector which is exposed to the photon beam is also an important factor in determining the effective area of the polarimeter. The primary purpose of the second detector, the calorimeter, is to aborb the full energy of the scattered photon.

The relative placement of the two detectors defines the scattering geometry. For incident photon energies below $100 \mathrm{keV}$, the azimuthal modulation of the scattered photons is maximized if the two detectors are placed at a right angle relative to the incident photon beam $\left(\theta=90^{\circ}\right.$; c.f., Fig. 1). The positioning of the two detectors must also be arranged relatively close to each other so that there is a finite solid angle for scattering to achieve the required detection efficiency. At the same time, a larger separation between the detectors provides more precise scattering geometry information. The accuracy with which the scattering geometry can be measured determines the ability to define the modulation pattern (Fig. 2) and therefore has a direct impact on the polarization sensitivity (see below). Here, one must compromise between total efficiency (small detector separation) and the ability to define the modulation pattern (large detector separation).

In principle, the scattering detector need not be active. At soft X-ray energies $(<10 \mathrm{keV})$, for example, the scattering process results in a very small energy loss that can be difficult to detect. For polarimeters designed to measure soft X-rays, the scattering element is usually passive, its only requirement being that it be designed to 
maximize the probability of a single photon scatter. At higher energies, an active element is certainly preferred as a means to minimize background, but it is certainly not required.

The ultimate goal of a Compton scatter polarimeter is to measure the azimuthal modulation pattern of the scattered photons. From equation (1), we see that the azimuthal modulation follows a $\cos 2 \eta$ distribution. More specifically, we can write the integrated azimuthal distribution of the scattered photons as,

$$
C(\eta)=A \cos \left(2\left(\eta-\phi+\frac{\pi}{2}\right)\right)+B
$$

where $\phi$ is the polarization angle of the incident photons. (In practice, a measured distribution must also be corrected for geometrical effects based on the corresponding distribution for an unpolarized beam. ${ }^{20}$ ) The quality of the polarization signal is quantified by the polarization modulation factor. ${ }^{20,23}$ For a given energy and incidence angle for an incoming photon beam, this can be expressed as,

$$
\mu_{p}=\frac{C_{p, \max }-C_{p, \min }}{C_{p, \max }+C_{p, \min }}=\frac{A_{p}}{B_{p}}
$$

where $C_{p, \max }$ and $C_{p, \min }$ refer to the maximum and minimum number of counts registered in the polarimeter, respectively, with respect to $\eta ; A_{p}$ and $B_{p}$ refer to the corresponding parameters in equation (4). In this case the $p$ subscript denotes that this refers to the measurement of a beam with unknown polarization. In order to determine the polarization of the measured beam, we need first to know how the polarimeter would respond to a similar beam, but with $100 \%$ polarization. This can be done using Monte Carlo simulations. We then define a corresponding modulation factor for an incident beam that is $100 \%$ polarized,

$$
\mu_{100}=\frac{C_{100, \text { max }}-C_{100, \text { min }}}{C_{100, \text { max }}+C_{100, \text { min }}}=\frac{A_{100}}{B_{100}}
$$

Then, following Novick, ${ }^{23}$ we can then use this result, in conjunction with the observed modulation factor $\left(\mu_{p}\right)$, to determine the level of polarization in a measured beam,

$$
P=\frac{\mu_{p}}{\mu_{100}}
$$

where $P$ is the measured polarization. The minimum detectable polarization (MDP) can be expressed as, ${ }^{23}$

$$
M D P(\%)=\frac{n_{\sigma}}{\mu_{100} S} \sqrt{\frac{2(S+B)}{T}}
$$

where $n_{\sigma}$ is the significance level (number of sigma), $S$ is the total source counting rate, $B$ is the total background counting rate and $T$ is the total observation time. Improved sensitivity to source polarization can be achieved either by increasing the modulation factor $\left(\mu_{100}\right)$ or by increasing the effective area of the polarimeter (thereby increasing the source counting rate, $S$ ).

\section{THE RHESSI SPECTROMETER}

The principle scientific objectives of RHESSI will be achieved with high resolution imaging spectroscopy observations from soft X-rays to gamma-rays. ${ }^{24}$ The imaging system is made up of nine Rotating Modulation Collimators (RMCs), each consisting of a pair of widely separated grids mounted on the rotating spacecraft. The spectrometer (Fig. 3) consists of nine segmented Germanium detectors, one behind each RMC, to detect photons from $3 \mathrm{keV}$ to $20 \mathrm{MeV} .^{25}$ These detectors represent the largest, readily available, hyperpure (n-type) coaxial Ge material $(\sim 7.1 \mathrm{~cm}$ diam $\times 8.5 \mathrm{~cm}$ long $)$. The detectors are cooled to $\sim 75^{\circ} \mathrm{K}$ by a space-qualified long-life mechanical cryocooler. Each detector (Fig. 4) is made from a single germanium crystal, which is electrically divided into independent front and rear segments to provide an optimum response for low and high 

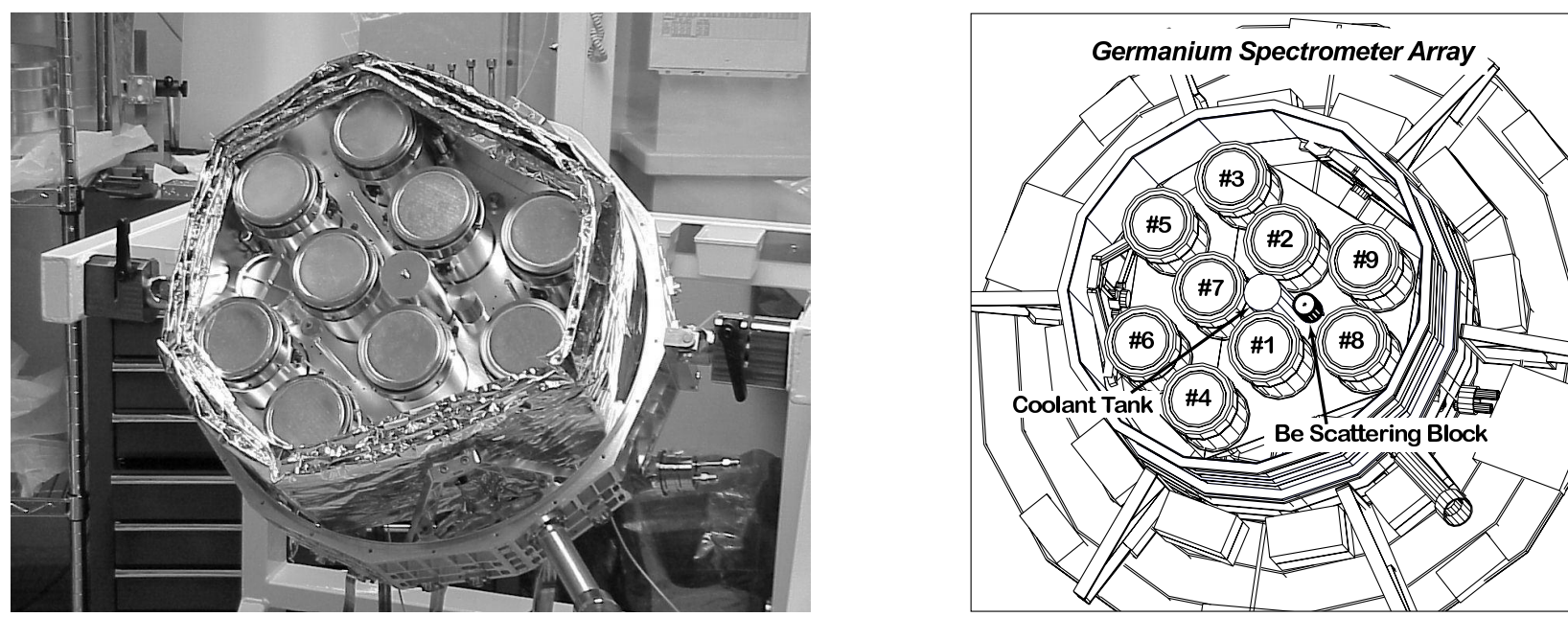

Figure 3: A pre-flight photo of the RHESSI spectrometer array (left) and a view of the same structure based on the RHESSI Mass model (right), showing the location of the Be scattering block.

energy photons, respectively. The inner electrode is segmented into two contacts that collect charge from two electrically independent detector segments, defined by the electric field pattern. This provides the equivalent of $\mathrm{a} \sim 1 \mathrm{~cm}$ thick planar Ge detector (the front segment) in front of a thick $\sim 7 \mathrm{~cm}$ coaxial Ge detector (the rear segment).

The front segment thickness is chosen to stop photons incident from the front (solar-facing side) of the instrument up to $\sim 100 \mathrm{keV}$, where photoelectric absorption dominates, while minimizing the active volume for background. Front-incident photons that Compton-scatter, and background photons or particles entering from the rear, are rejected by anticoincidence with the rear segment. A passive, graded-Z ring around the front segment (a laminate of $\mathrm{Ta} / \mathrm{Sn} / \mathrm{Fe}$ ) absorbs hard X-rays incident from the side, to provide the unusually low background of a phoswich-type scintillation detector.

Solar photons with energies from $\sim 100 \mathrm{keV}$ to $\sim 20 \mathrm{MeV}$, including all nuclear gamma-ray lines, stop primarily in the thick rear segment alone. A smaller fraction of these high energy photons will stop in the front segment after first Compton scattering in the rear segment, thus depositing energy in both the front and rear segments. Alternatively, some of these photons may deposit energy in two or more Ge detectors. The intense 3-100 keV X-ray fluxes that usually accompany large $\gamma$-ray line flares are absorbed by the front segment, so the rear segment will always count at moderate rates. Photons with energy above $20 \mathrm{keV}$ from non-solar sources can penetrate the thin aluminum cryostat wall from the side and also be detected by the Ge detector rear segments.

RHESSI is a spinning spacecraft, with a spin rate of $\sim 15 \mathrm{rpm}$. The high angular resolution imaging capability of RHESSI imposes severe requirements on the knowledge of the instrument orientation direction at any given time. Two spacecraft systems provide the necessary aspect solution. A solar aspect system provides knowledge of Sun center in pitch and yaw to 1.5 arcsec. A star scanner is used to sample the roll orientation at least once per rotation. Interpolation between measurements allows the roll orientation to be determined at intermediate times with the required accuracy. At least one (and typically seven) star(s) are detected each rotation. Measurements of only one star, averaged over a minute, allow the roll angle to be determined to 2.7 arcmin. As the spacecraft rotates, the RMCs convert the spatial information from the source into temporal modulation of the photon counting rates of the Ge detectors. The energy and arrival time of every photon, together with spacecraft orientation data, are recorded in the spacecraft's on-board 4-Gbyte solid-state memory (sized to hold all the data from the largest flare) and automatically telemetered to the ground within 48 hours. These data can then be used to generate X-ray/ $\gamma$-ray images with an angular resolution of $\sim 2$ arcseconds and a FoV of $\sim 1^{\circ}$. 

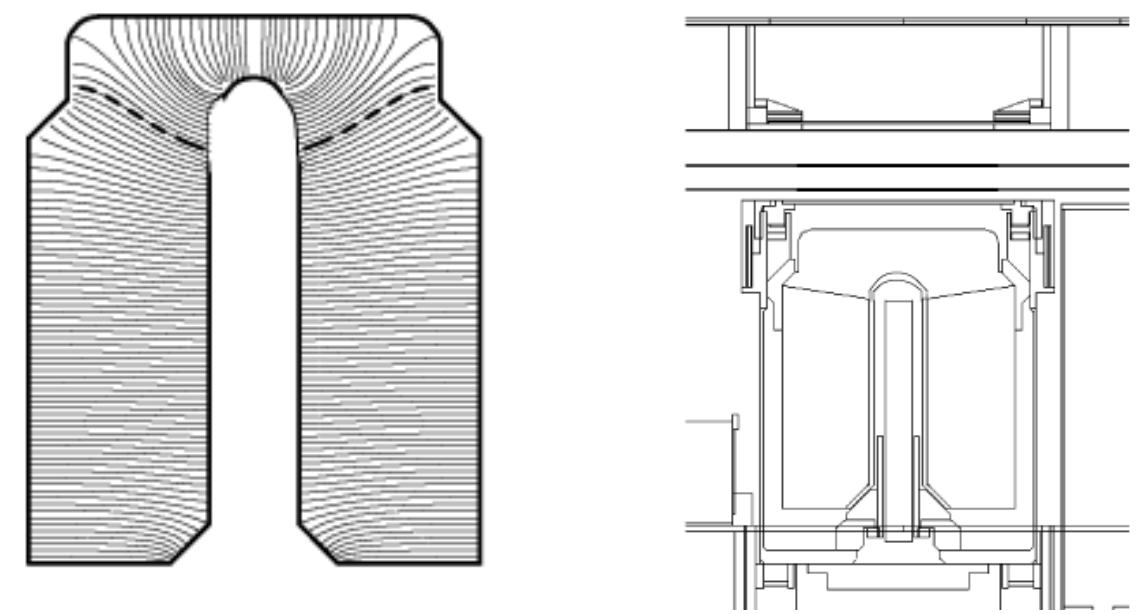

Figure 4: Cross section of a RHESSI detector. Left: A detector profile with field lines, the dashed field line indicating the separation between the front and back segments. Right: A detector in the cryostat, showing $\mathrm{Ta} / \mathrm{Sn} / \mathrm{Fe} / \mathrm{Al}$ shielding around the side of the front segment and above the shoulder of the rear segment.

\section{RHESSI AS A POLARIMETER}

It was realized during the RHESSI Phase-A study that the essential ingredients for measuring the polarization of solar hard X-rays, namely, an array of detectors in a rotating spacecraft, were already present in the RHESSI spectrometer. All that was needed was the addition of a strategically placed cylinder of beryllium in the cryostat to Compton scatter the solar flare hard X-rays $(20-100 \mathrm{keV})$ into the rear segments of the adjacent Ge detectors. For the purpose of making polarization measurements, the important feature of the specrometer design is that photons below $100 \mathrm{keV}$ that are detected in the rear segments are either non-solar back-ground or else are solar photons that scatter into the rear segments (e.g., from the Be scattering block). The distribution of events scattered into the adjacent Ge detector rear segments (with respect to some fixed reference frame) provides a polarization signature, since the direction of the scattering depends on the orientation of the electric field vectors, or plane of polarization, of the incoming photons. The spacecraft rotation provides a means to reduce the impact of systematic differences in detection efficiency amongst the different Ge detectors and to increase the sampling frequency with respect to the azimuthal scatter angle ( $\eta$ in Eq. 1).

The beryllium scattering block ( $3 \mathrm{~cm}$ in diameter by $3.5 \mathrm{~cm}$ long) was placed within the spectrometer cryostat, near the center of the Ge detector array (Fig. 3). Directly in front of the cryostat is a graded-Z shield (a laminate of $\mathrm{Ta} / \mathrm{Sn} / \mathrm{Fe}$ ) that is designed to absorb a large fraction of the flux below $100 \mathrm{keV}$, flux which tends to dominate the flare event. Openings in this shield provide an unattenuated path for these low energy photons from the Sun to reach the front surface of the cryostat directly in front of each Ge detector and directly in front of the Be scattering block. Thinned windows in the cryostat are designed to maximize the transmission of low energy solar photons to each Ge detector and to the Be scattering block without compromising the cryostat integrity. In addition, the trays which hold the RMC grids each have a hole directly in front of the Be scattering block. The total mass directly in front of the Be block amounts to $1 \mathrm{~mm}$ of aluminum in the cryostat plus some aluminized mylar thermal blankets, for a total mass of $2 \mathrm{~mm}$ aluminum equivalent.

The sensitive energy range for polarization measurements with RHESSI is about 20-100 keV. The graded$\mathrm{Z}$ shield in front of the cryostat insures that solar flare photons of this energy which enter the cryostat will interact either in the front surface of a Ge detector or in the Be block. The segmented design of each Ge detector further insures that there are no direct solar flare photons in the rear segments within the $20-100 \mathrm{keV}$ energy range. Flare photons within this energy range can only reach the rear segments by first scattering within the Be block. There is, however, a significant background from higher energy flare photons which scatter into the rear segments from other parts of the spacecraft and from flare photons which reflect off the Earths atmosphere (the 
Earth albedo flux). Photons that scatter into the Ge detectors from other parts of the spacecraft will generally not vary with spin angle. This unmodulated component will interfere with the polarization measurement by effectively reducing the modulation factor.

The albedo flux poses a potentially significant problem in that the level of albedo flux reaching a given Ge detector can vary significantly with spin phase (depending on the orientation of the spacecraft with respect to the local zenith). The magnitude of the albedo flux can be quite large, as much as $40-50 \%$ of the direct solar flare flux at energies below $100 \mathrm{keV}$. Fortunately, the albedo flux has only 1 maximum per spin period instead of the two maxima exhibited by the polarization signal and can therefore be recognized using an appropriate analysis.

The impact of the albedo flux may be somewhat further complicated by the fact that the precise distribution of the albedo flux across the visible disk of the Earth can also depend upon the polarization parameters of the direct flux from the flare. McConnell et al. ${ }^{26,27}$ described this effect and how it might be used to measure the (energy-averaged) polarization parameters of hard X-rays from solar flares or $\gamma$-ray bursts using the BATSE detectors on CGRO. While we expect that this effect will be quite difficult to observe with RHESSI, and hence unimportant in the polarization analysis, it is nontheless desirable to check this assumption with appropriate simulations. If, by some chance, this effect could be measured by RHESSI, it would provide a useful complement to the direct polarization measurement.

Finally, the variations in count rate within the detectors which measure the Be-scattered flux must be separated from intrinsic variations in the flare, but this can be done by examining the summed rate of the Ge detectors. Any variations in count rate that may be related to the source modulation by the grids happens much faster than a spin period, and should average out for the polarization measurements.

\section{SIMULATION OF THE RHESSI POLARIZATION RESPONSE}

To simulate the polarimetric response of RHESSI, we have used a modified version of the GEANT3 code that includes the effects of polarization in Compton scattering and tracks the polarization of the primary photon. The simulations make use of a detailed GEANT3 mass model that was developed by the RHESSI team. This is a comprehensive mass model that includes not only the RHESSI spectrometer, but also the complete telescope assembly, supporting electronics, the spacecraft support structure and the solar panels.

In principle, each of the nine Ge detectors provides a polarization signature. However, the quality of the signature varies, depending on the distance of the detector from the Be scattering block and the extent to which that detector is blocked from view with respect to the Be. A particular detector may be blocked from view by another detector and/or by a cryogenic coolant tank. (The coolant tank is used only for ground operations and is empty in flight.) Furthermore, the Ge detectors that are furthest from the Be do not provide a polarization signature with sufficient signal-to-noise to be useful in polarization studies. The Ge detectors that are most useful in polarization studies are detectors 1, 2, 8 and 9 (c.f., Figure 3). Unfortunately, detector 2 is currently not operating in segmentation mode and is therefore not usable for polarization studies. The simulations presented below assume that only detectors 1,8 and 9 are used.

The initial simulations involved directing a narrow mono-energetic beam directly at the Be block. In this case, the beam area corresponded to the area of the Be block $(3 \mathrm{~cm}$ diameter). These simulations allowed us to study the intrinsic polarimetric capabilities of RHESSI. A second set of simulations used a much broader beam (60 $\mathrm{cm}$ diameter) that covered the full area of the telescope tube assembly. These more realistic simulations allowed us to study the effects of photon scattering within the telescope tube and the surrounding spacecraft materials. In this case, photon scattering refers to the solar photons which interact in rear Ge segments after scattering from some spacecraft component other than the Be. These photons carry no polarization signature and serve only to increase the level of background.

Fig. 5 shows the simulated modulation patterns (number of counts versus azimuthal scatter angle) as derived from both the narrow and broad beam simulations. Only those events showing energy deposits above the 20 $\mathrm{keV}$ threshold in a single rear Ge segment (in detector 1, 8 or 9) are included. At lower energies (50 keV), the modulation patterns are very similar. But at higher energies $(80 \mathrm{keV})$, the broad beam simulation shows 

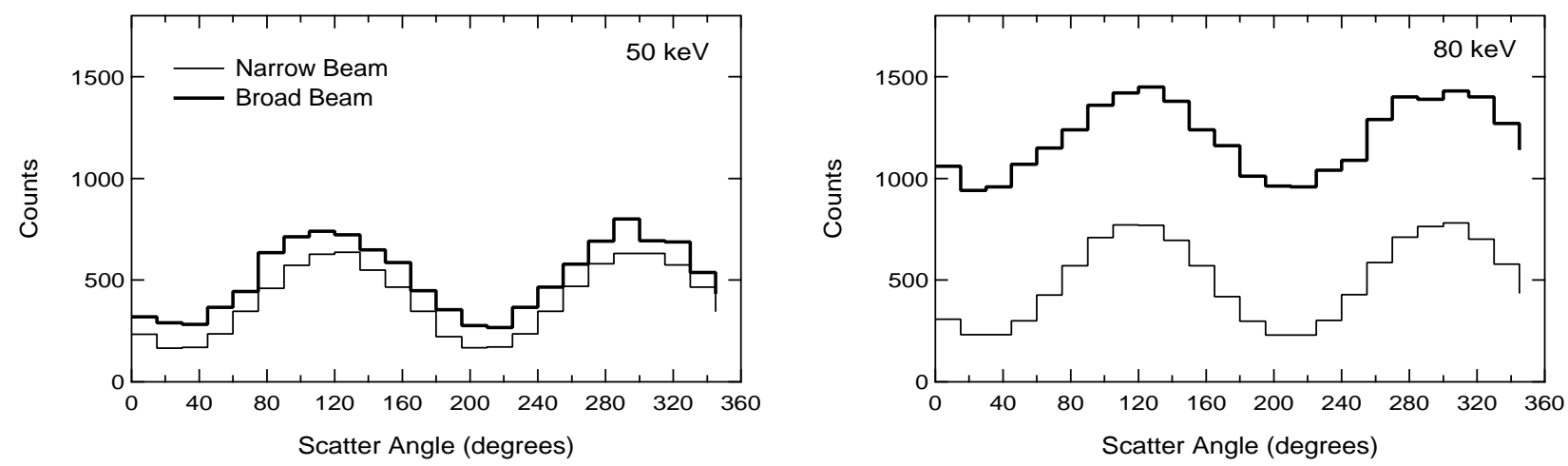

Figure 5: The modulation patterns produced from both narrow and broad beam simulations for $50 \mathrm{keV}$ (left) and $80 \mathrm{keV}$ (right). Although the total number of events increase dramatically at high energies using the more realistic broad beam simulation, most of these are unmodulated.

that many more photons are detected. Unfortunately, most of these photons are randomly scattered into the Ge detectors from material other than the Be. These additional events therefore carry no useful polarization signature. Fig. 6 shows the modulation patterns for three different energies (30, 50 and $80 \mathrm{keV}$ ), as derived from the broad beam simulations.

We characterize the polarization response of RHESSI using two parameters: 1) the effective area $\left(A_{e f f}\right)$, which represents the effective area for events satisfying the necessary criteria (single energy deposit in rear segments of the selected Ge detectors); and 2) the polarization modulation factor $\left(\mu_{100}\right)$, which can be thought of as a measure of the quality of the polarization signature (Eq. 6). These two quantities can be used to define a third parameter, the figure-of-merit, that is useful in characterizing the polarization response,

$$
F o M=\mu_{100} \sqrt{A_{\text {eff }}}
$$

As defined here, this figure-of-merit does not incorporate the effects of detector background. The characterization of the simulated response is shown in Figs. 7-9. As before, we include only those events that deposit energy above threshold $(>20$

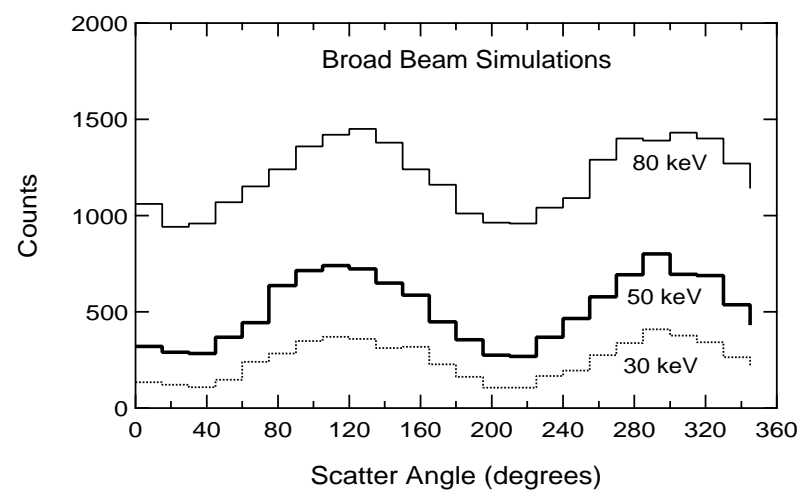

Figure 6: The modulation patterns derived from the more realistic broad beam simulations for three different energies $(30,50$ and $80 \mathrm{keV})$. As energy increases, the total number of detected events increases, but the modulation factor decreases as a result of scattered events.

$\mathrm{keV}$ ) in a single rear segment of detector 1,8 or 9. Of particular importance here is the significant impact of scattered photons, which is most easily seen in terms of the effective area (Fig. 7). The effective area is larger with the broad beam simulations as a result of the larger number of valid events due to photon scattering. Above about $50 \mathrm{keV}$, scattered photons become important. At energies near $100 \mathrm{keV}$ and above, scattering completely dominates the response. The effects of scattering are also seen in the plot of modulation factor versus energy (Fig. 8). Since the scattered component carries with it no polarization signature, the modulation factor decreases at energies above $\sim 30 \mathrm{keV}$, as scattering becomes more and more important. Fig. 9 shows that the efficacy of the HESSI polarimeter mode peaks near $60 \mathrm{keV}$ (although this definition does not incorporate the effects of an energy dependent background).

These results indicate that RHESSI is most effective as a polarimeter at energies below $\sim 100 \mathrm{keV}$. One can also see that having an active scattering element, which would have allowed for rejection of scattered photons 


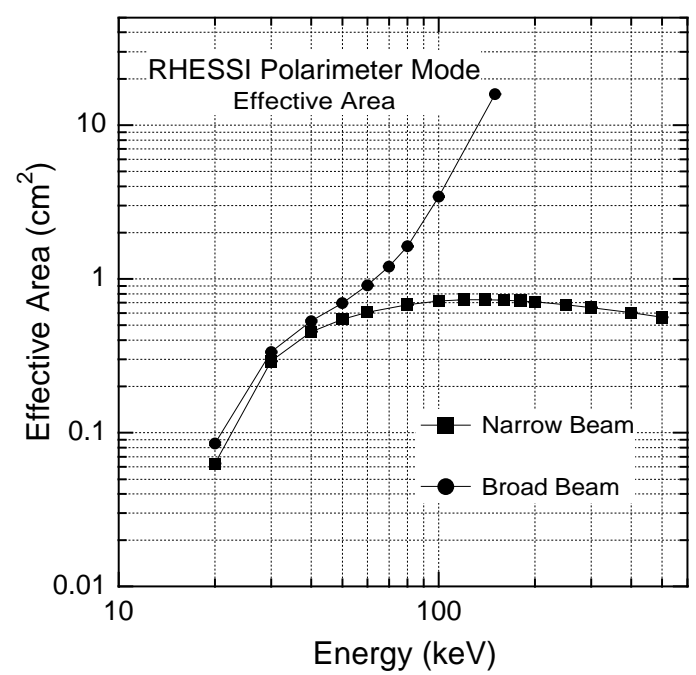

Figure 7: Effective area of the polarimetry mode as a function of energy for both the narrow beam and broad beam simulations. The effective area increases dramatically at higher energies, as a result of photons scattering into the Ge.

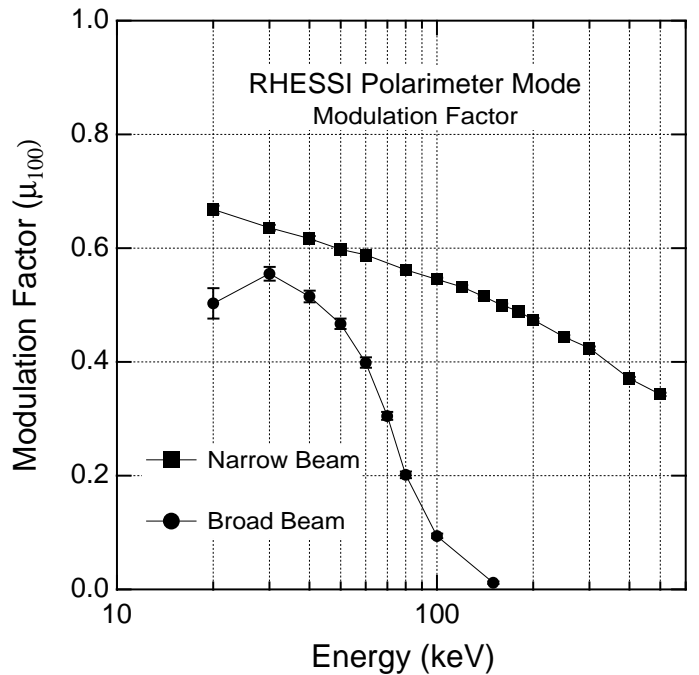

Figure 8: Modulation factor of the polarimetry mode as a function of energy for both the narrow beam and broad beam simulations. The modulation factor decreases dramatically at higher energies, as a result of unmodulated photons scattering into the Ge.

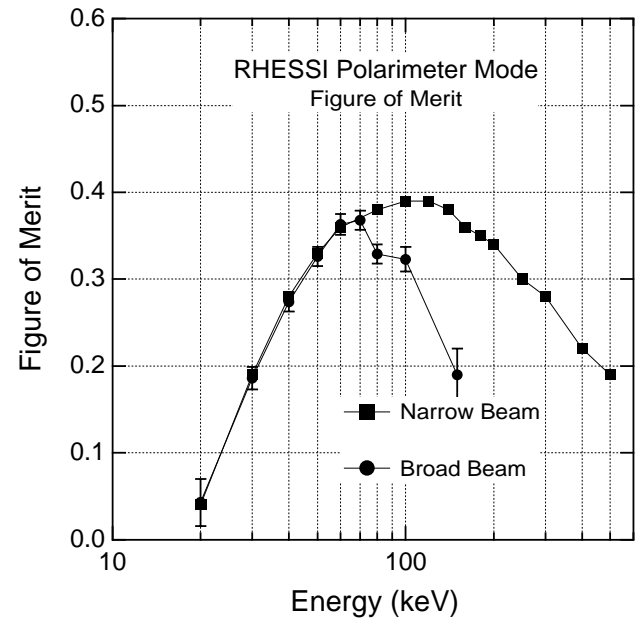

Figure 9: Figure of merit of the polarimetry mode as a function of energy for both the narrow beam and broad beam simulations. 


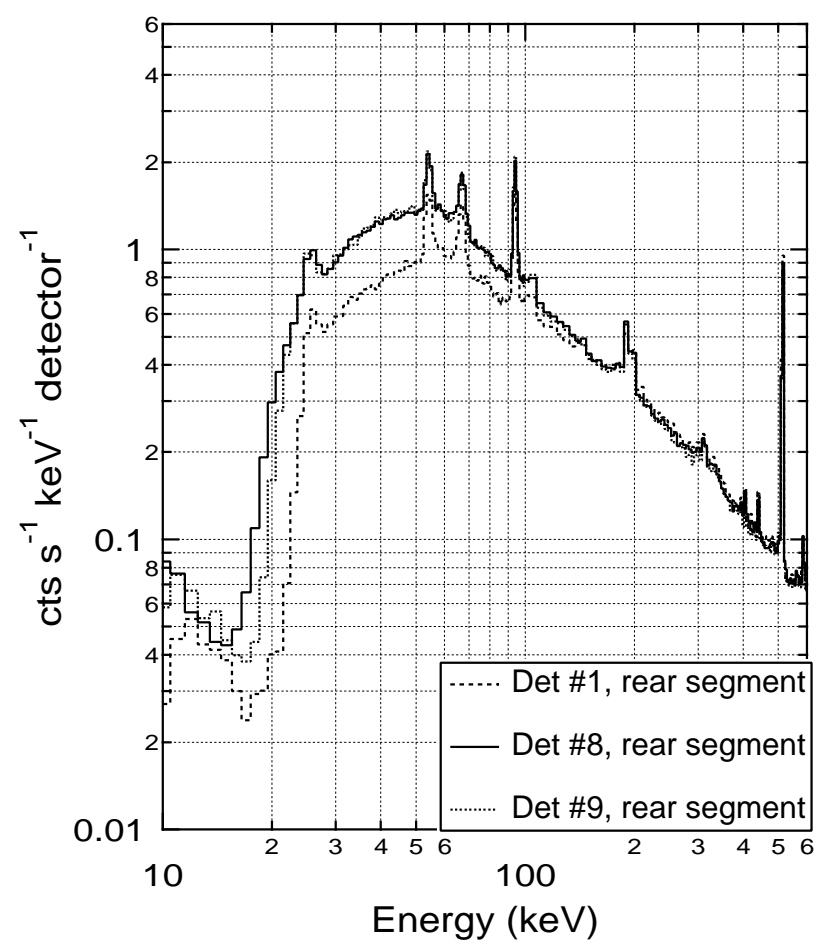

Figure 10: Measured background spectra for RHESSI detectors 1, 8 and 9.

by selecting on only those events scattered in the Be, would have provided a very significant improvement in the polarimetric capability of RHESSI.

\section{RHESSI SENSITIVITY TO SOLAR FLARE POLARIZATION}

An important component of any sensitivity estimate is the instrumental background. The measured RHESSI instrumental background is shown in Fig. 10. This spectrum, along with the simulated polarimetric characteristics, can be used to estimate the polarization sensitivity for a typical solar flare. Unfortunately, it is difficult to define a typical solar flare to use as a baseline for estimating polarization sensitivities. Here we have used an class X2 flare from Chanan, Emslie and Novick, ${ }^{10}$ with a spectrum of the form,

$$
\frac{d F}{d E}=3.6 \times 10^{7} E_{k e V}^{-4.3} \mathrm{~cm}^{-2} s^{-1} \mathrm{keV}^{-1}
$$

The X-ray classification depends only on the peak X-ray flux. Therefore, the polarization sensitivity for a given class flare will depend on the duration of the event. We have used durations ranging from 20 seconds up to 500 seconds, with an average spectrum corresponding to that given above. The results of these estimates are shown in Table 1. Results for both an X2 class flare and an X10 class flare are shown, with the X10 spectrum assumed to have the same form as that above, but with a factor of 5 increase in intensity. The polarization sensitivity is given as the minimum detectable polarization (MDP). The values represent a $3 \sigma$ sensitivity in terms of percent polarization.

These estimates indicate that RHESSI has a sufficient polarization sensitivity to measure the polarization of X-class flares down to a level of below $10 \%$ and, in some cases, below $1 \%$. This level of sensitivity will be useful in constraining various models that have been published in the literature.

Our sensitivity estimates have not yet considered the effects of albedo flux scattered from the Earths atmosphere. This may be significant and will likely degrade the sensitivity estimates given below. However, even if 
Table 1: Minimum Detectable Polarization (MDP)

\begin{tabular}{|c|c|c|c|}
\hline & \multicolumn{3}{|c|}{ Event Duration } \\
& $20 \mathrm{sec}$ & $100 \mathrm{sec}$ & $500 \mathrm{sec}$ \\
\hline X2 class flare & & & \\
$20-40 \mathrm{keV}$ & $11 \%$ & $5 \%$ & $2 \%$ \\
$40-60 \mathrm{keV}$ & $53 \%$ & $24 \%$ & $11 \%$ \\
$60-80 \mathrm{keV}$ & - & - & $46 \%$ \\
\hline X10 class flare & & & \\
$20-40 \mathrm{keV}$ & $5 \%$ & $2 \%$ & $1 \%$ \\
$40-60 \mathrm{keV}$ & $17 \%$ & $7 \%$ & $3 \%$ \\
$60-80 \mathrm{keV}$ & $61 \%$ & $27 \%$ & $12 \%$ \\
\hline
\end{tabular}

this component is comparable to the intrinsic detector background (as might be expected), the quoted sensitivities might be about 50\% worse than shown. Even such a degraded sensitivity will still provide important scientific results.

\section{PROSPECTS FOR SOLAR FLARE POLARIZATION STUDIES}

The RHESSI spacecraft was successfully launched on February 5, 2002. To date, there have been several X-class solar flares that are good candidates for polarization studies. We are currently working on the detailed analysis of these data. This is a difficult analysis, but one that is sure to provide interesting results.

\section{ACKNOWLEDGMENTS}

This work has been supported by NASA grant NAG5-10203 at UNH and UAH, and contract NAS5-98033 at UC Berkeley.

\section{REFERENCES}

1. M. L. McConnell, D. M. Smith, A. G. Emslie, R. P. Lin, and J. M. Ryan, "Prospects for hard X-ray solar flare polarimetry with HESSI," to be published in Multi-Wavelength Observations of Coronal Structure and Dynamics - Yohkoh Tenth Anniversary Meeting, Elsevier, Oxford, 2002.

2. T. Bai and R. Ramaty, "Backscatter, anisotropy, and polarization of solar hard X-rays," Ap. J. 219, pp. 705-726, 1978.

3. J. C. Brown, "The directivity and polarisation of thick target X-ray bremsstrahlung from solar flares," Solar Phys. 26, pp. 441-459, 1972.

4. Ju. E. Charikov, A. B. Guzman, and I. V. Kudryavtsev, "Hard X-ray emission of solar flares and nonstationary kinetics of electron beams," Astron. Astrophys. 308, pp. 924-928, 1996.

5. A. G. Emslie and L. Vlahos, "Radiation signatures from a locally energized flaring loop," Ap. J. 242, pp. 359-373, 1980.

6. S. H. Langer and V. Petrosian, "Impulsive solar X-ray bursts. III - Polarization, directivity, and spectrum of the reflected and total bremsstrahlung radiation from a beam of electrons directed toward the photosphere," Ap. J. 215, pp. 666-676, 1977.

7. J. Leach and V. Petrosian, "The impulsive phase of solar flares. II - Characteristics of the hard X-rays," Ap. J. 269, pp. 715-727, 1983. 
8. V. V. Zharkova, J. C. Brown, and D. V. Syniavskii, "Electron beam dynamics and hard X-ray bremsstrahlung polarization in a flaring loop with return current and converging magnetic field," Astron. Astrophys. 304, pp. 284-295, 1995.

9. A. G. Emslie and J. C. Brown, "The polarization and directivity of solar-flare hard X-ray bremsstrahlung from a thermal source," Ap. J. 237, pp. 1015-1023, 1980.

10. G. Chanan, A.G. Emslie, and R. Novick, "Prospects for solar flare X-ray polarimetry," Solar Phys. 118, pp. 309-319, 1988.

11. S. Masuda, T. Kosugi, H. Hara, T. Sakao, K. Shibata, and S. Tsuneta, "Hard X-ray sources and the primary energy-release site in solar flares," Publ. Astron. Soc. Japan 47, pp. 677-689, 1995.

12. I. P. Tindo, V. D. Ivanov, S. L. Mandel'stam, and A. I. Shuryghin, "On the Polarization of the Emission of X-Ray Solar Flares," Solar Phys. 14, pp. 204-207, 1970.

13. I. P. Tindo, V. D. Ivanov, B. Valnicek, and M. A. Livshits, "Preliminary interpretation of the polarization measurements performed on 'Intercosmos-4' during three X-ray solar flares," Solar Phys. 27, pp. 426-435, 1972.

14. I. P. Tindo, V. D. Ivanov, S. L. Mandel'stam, and A. I. Shuryghin, "New measurements of the polarization of X-ray solar flares," Solar Phys. 24, pp. 429-433, 1972.

15. J. C. Brown, A. N. McClymont, and I. S. McLean, "Interpretation of solar hard X-ray burst polarisation measurements," Nature 247, pp. 448-449, 1974.

16. M. P. Nakada, W. M. Neupert, and R. J. Thomas, "Polarization results of solar X-rays from OSO-7," Solar Phys. 37, pp. 429-435, 1974.

17. I. P. Tindo, A. I. Shuryghin, and W. Steffen, "The polarization of X-ray emission of some solar flares in July 1974," Solar Phys. 46, pp. 219-227, 1976.

18. J. R. Lemen, et al., "A solar flare X-ray polarimeter for the space shuttle," Solar Phys. 80, pp. 333-349, 1980.

19. L. J. Tramiel, G. A. Channan, R. and Novick, "Polarization evidence for the isotropy of electrons responsible for the production of 5-20 keV X-rays in solar flares," Ap. J. 280, pp. 440-447, 1984.

20. F. Lei, A.J. Dean and G.L. Hills, "Compton scatter polarimetry in gamma-ray astronomy," Space Sci. Rev. 82, pp. 309-388, 1997.

21. R. D. Evans, The Atomic Nucleus, McGraw-Hill, New York, 1958.

22. E. L. Chupp, Gamma-Ray Astronomy, D. Reidel Publishing Co., Boston, 1976.

23. R. Novick, "Stellar and solar X-ray polarimetry," Space Sci. Rev. 18, pp. 389-408, 1975.

24. R. P. Lin et al., "The High Energy Solar Spectroscopic Imager (HESSI) Mission," in ASP Conf. Ser. 206, High Energy Solar Physics - Anticipating HESSI, R. Ramaty and N. Mandzhavidze, ed., pp. 1-12, ASP, San Francisco, 2000.

25. D. M. Smith et al., "The HESSI Spectrometer," in ASP Conf. Ser. 206, High Energy Solar Physics Anticipating HESSI, R. Ramaty and N. Mandzhavidze, ed., pp. 92-101, ASP, San Francisco, 2000.

26. M. McConnell, et al., "Hard X-ray polarimetry of solar flares with BATSE," in AIP Conf. Proc. 374, High Energy Solar Physics, R. Ramaty, N. Mandzhavidze and X.-M. Hua, ed., pp. 368-372, AIP, New York, 1996.

27. M. McConnell, et al., "Using BATSE to measure gamma-ray burst polarization," in AIP Conf. Proc. 384, Gamma Ray Bursts, 3rd Huntsville Symposium, C. Kouveliotou, M. F. Briggs and G. J. Fishman, ed., pp. 851-855, AIP, New York, 1996. 